\title{
AIRE deficiency in thymus of 2 patients with Omenn syndrome
}

\author{
Patrizia Cavadini, ${ }^{1}$ William Vermi, ${ }^{2}$ Fabio Facchetti, ${ }^{2}$ Stefania Fontana, ${ }^{1}$ Seiho Nagafuchi, ${ }^{3}$ \\ Evelina Mazzolari, ${ }^{1}$ Anna Sediva, ${ }^{4}$ Veronica Marrella, ${ }^{5}$ Anna Villa, ${ }^{5}$ Alain Fischer, ${ }^{6}$ \\ Luigi D. Notarangelo, ${ }^{1}$ and Raffaele Badolato ${ }^{1}$
}

\begin{abstract}
${ }^{1}$ Istituto di Medicina Molecolare "Angelo Nocivelli," Clinica Pediatrica, and ${ }^{2}$ Cattedra di Anatomia Patologica, University of Brescia, Brescia, Italy. ${ }^{3}$ Laboratory of Molecular Immunology and Diabetes, School of Medicine, Kyushu University, Fukuoka, Japan. ${ }^{4}$ Institute of Immunology, University Hospital Motol, Prague, Czech Republic. 5 Istituto Tecnologie Biomediche, Consiglio Nazionale delle Ricerche, Milan, Italy. 6INSERM U429, Necker-Enfants Malades, Paris, France.
\end{abstract}

\begin{abstract}
Omenn syndrome is a severe primary immunodeficiency with putative autoimmune manifestations of the skin and gastrointestinal tract. The disease is caused by hypomorphic mutations in recombination-activating genes that impair but do not abolish the process of VDJ recombination, leading to the generation of autoreactive $T$ cells with a highly restricted receptor repertoire. Loss of central tolerance in genetically determined autoimmune diseases, e.g., autoimmune polyendocrinopathy-candidiasis-ectodermal dystrophy, is associated with defective expression by medullary thymic epithelial cells of AIRE, the transcription activator that induces thymic expression of tissue-specific antigens. Analysis of AIRE expression in the thymi of 2 Omenn syndrome patients and 1 SCID patient, by real-time RT-PCR and immunohistochemistry, demonstrated a profound reduction in the levels of AIRE mRNA and protein in patients as compared with a normal control subject. Lack of AIRE was associated with normal or even increased levels of keratin and lymphotoxin- $\beta$ receptor mRNAs, while mRNAs of the self-antigens insulin, cytochrome P450 1a2, and fatty acid-binding protein were undetectable in thymi from immunodeficiency patients. These results demonstrate that deficiency of AIRE expression is observed in severe immunodeficiencies characterized by abnormal $\mathrm{T}$ cell development and suggest that in Omenn syndrome, the few residual $T$ cell clones that develop may escape negative selection and thereafter expand in the periphery, causing massive autoimmune reactions.
\end{abstract}

\section{Introduction}

Omenn syndrome (OMIM 603554) is a rare autosomal recessive combined immune deficiency characterized by early-onset generalized erythroderma, failure to thrive, protracted diarrhea, hepatosplenomegaly, and lymphadenopathy, with elevated serum IgE and low serum Igs (1-3). In patients with Omenn syndrome, B cells are usually absent both in peripheral blood and in lymphoid tissues $(3,4)$, whereas there is an oligoclonal $\mathrm{T}$ cell infiltration of skin, gut, liver, and spleen of possible autoimmune origin (4-9).

Omenn syndrome is associated with a severe disturbance in both $\mathrm{T}$ and $\mathrm{B}$ cell development. Recombinase-activating genes (RAGs) 1 and 2 encode for 2 lymphoid proteins that are critically involved in the initiation of the $V(D) J$ recombination process. Null mutations of either RAG1 or RAG2 loci cause a profound block in $\mathrm{T}$ and $\mathrm{B}$ cell development and are thereafter responsible for a $\mathrm{T}^{-} \mathrm{B}^{-}$form of severe combined immunodeficiencies ( $\left.\mathrm{T}^{-} \mathrm{B}^{-} \mathrm{SCID}\right)$ (10). In contrast, we have previously shown that hypomorphic mutations at the RAG1 or RAG2 loci may allow for residual V(D)J recombination activity and cause Omenn syndrome (11). In Omenn syndrome patients, oligoclonal T cells with a highly restricted receptor repertoire are generated; these cells infiltrate and expand in peripheral tissues, including the skin and the gastrointestinal tract $(9,12)$. In a recent publication it has been hypothesized by

Nonstandard abbreviations used: AIRE, autoimmune regulator element; FABP, fatty acid-binding protein; LT- $\alpha$, lymphotoxin- $\alpha$; LT $\beta$ R, lymphotoxin- $\beta$ receptor; Pt, patient; RAG, recombinase-activating gene; TEC, thymic epithelial cell. Conflict of interest: The authors have declared that no conflict of interest exists.

Citation for this article: J. Clin. Invest. 115:728-732 (2005)

doi:10.1172/JCI200523087. one of us that autoimmune manifestations of Omenn syndrome might be related to loss of central tolerance (13), but the underlying mechanism has remained obscure.

Autoimmune regulator element (AIRE) is a transcriptional activator expressed by medullary thymic epithelial cells (TECs). Mutations of AIRE cause autoimmune polyendocrinopathycandidiasis-ectodermal dystrophy (APECED), a monogenic disease with autoimmune manifestations that affect the endocrine glands and eccrine tissues (14-16). Evidence from experimental animals indicates that AIRE expression in the thymus requires both a normal $\mathrm{T}$ cell development and a thymic structure with a well-organized corticomedullary architecture. In keeping with this, mice transgenic for the CD $3 \varepsilon$ chain, which present an early and severe block in thymocyte development at the stage of triple-negative thymocytes, fail to express AIRE (17). Moreover, defective AIRE expression and increased autoantibody production are observed in lymphotoxin- $\alpha$-null (LT- $\alpha-$ null) and in lymphotoxin- $\beta$ receptor-null (LT $\beta R$-null) mice (18). Finally, targeting of the RelB gene, which encodes for a component of the NF- $\mathrm{KB}$ transcription factor, results in profoundly disturbed thymic structure associated with impaired AIRE expression (19).

Recently, Anderson and colleagues have shown in a murine model that AIRE regulates the ectopic expression in the thymus of a set of tissue-specific proteins (e.g., insulin) that are normally found in the periphery; this suggests that AIRE is a key factor of central tolerance (20). In keeping with these observations, Goodnow and coworkers have shown that Aire-null mice are unable to delete pancreatic islet-reactive $\mathrm{T}$ cells in the thymus. AIRE is thus essential for mediating a highly efficient and specialized mechanism of deletion of thymic T cells (21). Since patients with Omenn 

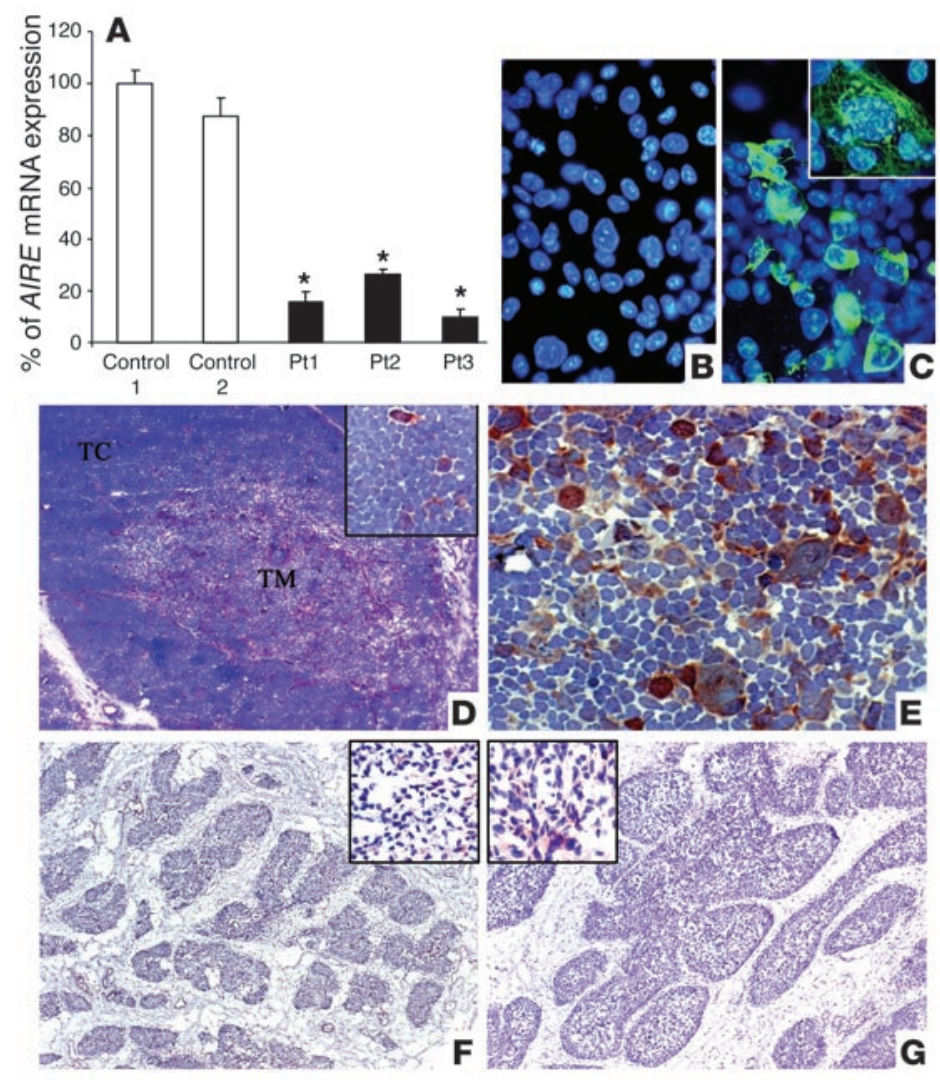

\section{Figure 1}

AIRE expression in thymi from Omenn syndrome and SCID patients. (A) Real-time PCR analysis of cDNA prepared from RNA isolated from normal thymi and from thymi of Omenn syndrome patients (Pt1 and Pt2) and 1 SCID patient (Pt3). The levels of AIRE mRNA were calculated as a percentage of those in normal thymus from control subject 1 and are expressed as average of triplicates \pm SE. Data are representative of 1 of 5 experiments. ${ }^{*} P<0.05$ compared with controls. (B-G) AIRE protein was detectable in AIRE-transfected cells (C) with the typical cytoplasmic microtubular pattern and multiple nuclear dots (C, inset), but not in mock-transfected cells (B). In normal thymus, a clear corticomedullary differentiation with numerous thymocytes was identified (D). AIRE protein was predominantly expressed in the medulla (TM), whereas only scattered positive cells with large and irregular morphology (D, inset) were found in the cortex (TC). A high magnification of the thymic medulla illustrates cytoplasmic and nuclear expression of AIRE by numerous large irregular cells (E). Thymus sections from a SCID patient (F) and an Omenn syndrome patient $(\mathbf{G})$ showed lymphoid depletion along with loss of corticomedullary differentiation and lack of AIRE protein expression ( $\mathbf{F}$ and $\mathbf{G}$, and insets). AIRE protein was detected by immunofluorescence (green fluorescence in $\mathbf{B}$ and $\mathbf{C}$ ) and by immunoperoxidase technique and counterstaining with Mayer's hematoxylin (D-G). Magnifications: ×100 (D, F, and G); ×200 (D, $\mathbf{F}$, and $\mathbf{G}$, insets); $\times 400$ (B, C, and E); $\times 1,000$ (C, inset). syndrome have a low number of $\mathrm{T}$ cell precursors with a productive $\mathrm{T}$ cell receptor rearrangement, we hypothesized that the reduced generation of thymic $T$ cells in this disease may result in low expression of AIRE. Consequently, the few autoreactive T cells that differentiate in the thymus could escape negative selection and expand in the periphery, thereby leading to the autoimmune manifestations of the Omenn syndrome. We have evaluated this hypothesis by analyzing expression of AIRE and of tissue-specific transcripts in thymic tissues obtained from 3 immunodeficiency patients with $R A G$ gene mutations ( 2 Omenn syndrome patients and 1 SCID patient).

\section{Results}

AIRE deficiency in thymi of Omenn syndrome patients. We investigated the expression of AIRE in the thymus and its regulation in 3 patients (Pts) with severe primary immunodeficiency due to mutations in RAGs, namely Omenn syndrome (Pt1 and $\mathrm{Pt} 2$ ) and $\mathrm{T}^{-} \mathrm{B}^{-}$ SCID (Pt3). Quantitative analysis of AIRE mRNA expression by real-time PCR showed that AIRE levels were significantly reduced in the thymi of the 2 Omenn syndrome patients and the T-B- SCID patient $(P<0.05)$ and constituted $10-25 \%$ of mRNA levels detected in 2 control thymi, thus suggesting a severe defect of AIRE expression in these patients (Figure 1A).

We next evaluated tissue expression and distribution of AIRE by single-staining immunohistochemistry using a rabbit antiAIRE polyclonal antibody (22). In AIRE-transfected COS7 cells, AIRE staining can be recognized as being either cytoplasmic (diffuse microtubular staining) or of a nuclear-dot pattern (23), whereas the polyclonal antibody did not react with mocktransfected cells (Figure 1, B and C).
On tissue sections from a control thymus, $\mathrm{AIRE}^{+}$cells were predominant in the medulla, with only scattered cells in the cortex (Figure 1D). High-power-field magnification of thymic medulla demonstrates that AIRE protein can be identified in the cytoplasm as well as in the nucleus (Figure 1E). Conversely, AIRE protein expression was markedly reduced on thymus sections from an Omenn syndrome patient ( $\mathrm{Pt} 1$ ) and a $\mathrm{T}^{-} \mathrm{B}^{-}$SCID patient (Pt3) (Figure 1, F and G).

Normal keratin expression in thymi of Omenn syndrome patients. Double-immunofluorescence staining with anti-AIRE and anti-keratin showed that in normal thymus, AIRE is expressed by a fraction of keratin ${ }^{+}$TECs as well as by large irregular cells that do not coexpress keratin. As previously reported, the latter population is likely to represent mature DCs (24) (Figure 2A). Thymus sections from patients with Omenn syndrome (Figure $2 \mathrm{~B}$ ) or T-B- SCID (data not shown) were nicely decorated by anti-keratin antibody but did not show reactivity with anti-AIRE antiserum. Accordingly, quantitation of keratin-13 mRNA levels, as analyzed by real-time PCR, showed a 2- to 4-fold increase of keratin-13 expression in thymi from the 2 Omenn syndrome patients in comparison with the control thymi, but the difference was not statistically significant (Figure 2C). This increased expression of keratin is likely to reflect the relative abundance of thymic epithelium and the depletion of the lymphoid compartment in Omenn syndrome.

Lack of insulin, cytochrome P450 1a2, and fatty acid-binding protein transcripts in thymi of Omenn syndrome patients. The interaction of developing thymocytes with medullary epithelial cells is crucial for induction of tissue-specific antigens by medullary epithelial cells and for negative selection of autoreactive T cell clones (20). LT- $\alpha$, which is expressed by thymocytes, participates in this interaction by activating murine medullary thymus epithelial cells through LT $\beta$ R. This 

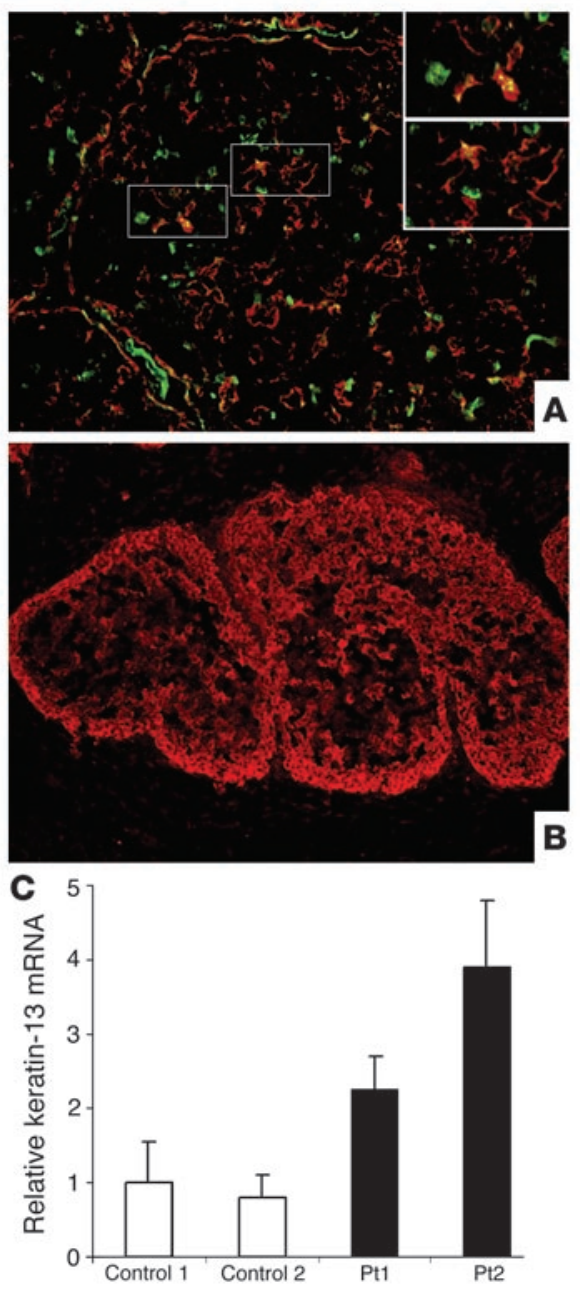

interaction is required for induction of AIRE expression by TECs (18, $25)$, although the molecular mechanisms involved remain largely unknown. Analysis of $L T \beta R$ mRNA expression showed that mRNA levels were higher in thymi of both Omenn syndrome patients as compared with controls (Table 1). The higher levels of $L T \beta R$ mRNAs in immunodeficiency patients are likely to reflect the relative abundance of the stromal compartment in the thymi of the patients.

Given the low levels of AIRE expression in thymi of the Omenn syndrome patients and the T-B-SCID patient, we evaluated the thymic expression of 3 transcripts encoding for the antigens insulin and cytochrome P450 1a2 and for the intestine-specific antigen fatty acid-binding protein (FABP). Insulin, cytochrome P450 1a2, and FABP mRNAs were found to be undetectable or severely reduced by real-time PCR analysis in thymi of the Omenn syndrome patients and the SCID patient, as compared with control thymi (Table 1). Since the process of negative selection of thymocytes is dependent on expression of trace amounts of tissue-specific antigens by medullary epithelial cells (20), these data support the hypothesis that defective thymic AIRE expression in Omenn syndrome can result in an impairment of thymic negative selection.

\section{Discussion}

AIRE expression in the thymus requires cross-talk between developing thymocytes and stromal cells. This process is dependent on an organized thymic microenvironment with an appropriate corti-

\section{Figure 2}

TECs in thymus from an Omenn syndrome patient. (A and B) Double fluorescence staining in thymus sections obtained from a control subject (A) and an Omenn syndrome patient (B). (A) In normal thymus, TECs were regularly distributed; a fraction of them showed large and irregular morphology and expressed AIRE. Insets represent high magnification of the corresponding rectangular area. (B) Conversely, thymus sections from an Omenn syndrome patient (Pt1) revealed a dense framework of keratin+ TECs, which did not react with anti-AIRE. Double-immunofluorescence analysis was performed with anti-AIRE (green in $\mathbf{A}$ and $\mathbf{B}$ ) and anti-keratin (red in $\mathbf{A}$ and $\mathbf{B}$ ). Magnifications: $\times 100$ (B), $\times 200$ (A), ×1,000 (A, insets). (C) Real-time PCR analysis of cDNA prepared from RNA isolated from whole thymus of 2 control subjects and 2 Omenn syndrome patients (Pt1 and Pt2). The levels of keratin-13 mRNA were calculated as fold increase over those in the control and are expressed as average of triplicates \pm SE. For each experiment, levels of keratin were normalized to levels of GAPDH mRNA. Data are representative of 1 of 4 experiments.

comedullary differentiation and normal representation of medullary epithelium (17). While epithelial cells are present in normal or even increased numbers in the thymi from the Omenn syndrome patients, we have shown that the typical compartmentalization of the thymus is missing in both Omenn syndrome and $\mathrm{T}^{-} \mathrm{B}^{-} \mathrm{SCID}$ thymi, which suggests that defective development of thymocytes due to $R A G$ gene mutations leads to loss of interaction between epithelial cells and thymocytes. RAG-null mice, which present a delayed defect of thymopoiesis at the stage of triple-negative CD $44^{-} \mathrm{CD} 25^{+}$thymocytes, present a typical architectural organization and normal AIRE expression, which suggests that clinical and immunological manifestations of $\mathrm{T}^{-} \mathrm{B}^{-}$SCID patients are distinct from the phenotype of RAG-null mice (17). Indeed, the SCID patient that we have reported presented a residual number of $\mathrm{T}$ cells, which reinforces the concept that, in humans, RAG mutations may lead to heterogeneous immune and clinical manifestations ranging from the complete absence of T cells that is observed in typical T-B- SCID to Omenn syndrome (26).

We speculate that the severe defect of AIRE expression in thymi of Omenn syndrome and SCID patients may account for the observed lack of tissue-specific antigens, such as insulin, cytochrome P450 $1 \mathrm{a} 2$, and FABP. In this setting, the few autoreactive T cells that develop in Omenn syndrome escape negative selection and expand in the periphery, leading to the typical manifestations of the disease. This hypothesis is supported by a recent observation that partial AIRE expression in thymus results in a dose-dependent expression of the insulin promoter in epithelial cells and in parallel clonal deletion of islet-reactive $\mathrm{T}$ cells, suggesting that even partial reduction may lead to organ-specific autoimmune manifestations (27).

There is accumulating evidence that in addition to central tolerance, self tolerance is maintained by a population of $\mathrm{CD} 4^{+} \mathrm{T}$ cells that express the transcription repressor gene Foxp3. It has been postulated that regulatory $\mathrm{CD}^{+} \mathrm{T}$ cells of thymic origin, upon encounter with self antigens in peripheral tissue, may actively suppress autoimmune reactions (28). It is possible that in addition to impaired expression of AIRE and of tissue-specific antigens, generation of thymic regulatory $\mathrm{CD}^{+} \mathrm{T}$ cells might also be affected in Omenn syndrome, so that several mechanisms may concur to the development of autoimmune manifestations in this immunodeficiency.

Autoimmune manifestations are also observed in other immunodeficiencies that are characterized by abnormal maturation of 


\section{Table 1}

Thymic expression of LT $\beta R$ and of tissue-specific antigens

\begin{tabular}{lcccc} 
LT $\beta \mathbf{R}$ & Insulin & $\begin{array}{c}\text { Cytochrome } \\
\text { P450 1a2 }\end{array}$ & FABP \\
Control 1 & 1 & 1 & 1 & ND \\
Control 2 & 0.91 & 0.8 & 0.88 & 1 \\
Pt1 & 217 & $<0.05$ & $<0.05$ & ND \\
Pt2 & 77 & $<0.05$ & $<0.05$ & 0.14 \\
Pt3 & 269 & $<0.05$ & $<0.05$ & ND \\
\hline
\end{tabular}

Real-time PCR analysis of cDNA prepared from RNA isolated from whole thymus of 2 control subjects, 2 Omenn syndrome patients (Pt1 and Pt2), and a SCID patient (Pt3). The fold increase of lymphotoxin- $\beta$ receptor (LT $\beta R$ ), insulin, cytochrome P450 1a2, and FABP mRNAs in patients was calculated relative to those of a normal thymus and expressed as average of triplicates. Data are representative of 1 of 2 experiments. ND, not done: insufficient sample to perform the assay.

thymocytes with residual nonfunctional circulating $\mathrm{T}$ cells, as observed in IL-2 receptor $\alpha$ chain deficiency, and in some leaky forms of $\mathrm{T}^{-} \mathrm{B}^{+} \mathrm{SCID}$ caused by mutations of JAK3 or IL2RG genes $(29,30)$. While the contributory role of hypothetical defects of central tolerance in these immunodeficiencies remains to be assessed, we hypothesize that Omenn syndrome may constitute a paradigmatic model of pathogenesis for more common autoimmune diseases associated with impaired $\mathrm{T}$ cell differentiation.

\section{Methods}

Patients. The case of the Omenn syndrome patient identified as Pt1 has been previously reported (9). Briefly, this 2-month-old girl, born from consanguineous parents, presented with failure to thrive, diarrhea, erythroderma, hypoproteinemia, and low serum Igs except for increased serum IgE level $\left(9.1 \times 10^{3} \mathrm{kU} / \mathrm{l}\right)$. Lymphocytes $\left(8.6 \times 10^{9}\right.$ cells per liter $)$ were mostly constituted by activated memory $\mathrm{T}$ cells with absence of $B$ cells and displayed an impaired proliferative response to mitogens. RAG2 genetic analysis demonstrated a homozygous missense mutation $(\mathrm{G} \rightarrow \mathrm{A})$ at nucleotide 1,887 , leading to substitution of glutamine for arginine at codon 229 (26). The second patient with Omenn syndrome (Pt2) was a girl born from non-consanguineous parents, without other affected family members, who presented at 3 months with oral thrush, axillary lymphadenopathy, and subcutaneous abscesses at the site of Bacille Calmette-Guérin vaccination. After 3 months, the patient developed disseminated CMV infection and died of untreatable respiratory failure. Lymphocytes were $3.9 \times 10^{9}$ cells per liter with prevalence of activated memory T cells (CD3, 32\%; CD4, 30\%; CD8, $1.2 \%$; TCR $\alpha / \beta$, 29\%; HLA-DR, 12\%; CD16, 67\%; CD45RO/CD4, 99\%) and depletion of B cells (CD19, 0.6\%). Ig levels were as follows: IgG, $5.12 \mathrm{~g} / 1$; IgA, $0.07 \mathrm{~g} / \mathrm{l}$; IgM, $0.18 \mathrm{kU} / 1$. Genetic analysis showed a compound heterozygosity at the RAG1 locus, with deletion of dinucleotides 368-369 and a singlenucleotide mutation (C2258T), resulting in the arginine-to-tryptophan amino acid change at position 716. The dinucleotide 368-369 mutation was previously reported and characterized in another patient (31).

The SCID patient (Pt3) was a girl who presented at 3 months with fever, anemia, failure to thrive, and intractable diarrhea. Because of anemia (hemoglobin, $7.3 \mathrm{~g} / \mathrm{dl}$ ), she received a blood transfusion. Laboratory examinations showed lymphopenia $\left(0.88 \times 10^{3}\right.$ cells per microliter $)$, presence of NK cells but not of B cells ( $\left.\mathrm{T}^{-} \mathrm{B}^{-} \mathrm{NK}^{+} \mathrm{SCID}\right)$, and engraftment of allogeneic $\mathrm{T}$ cells derived from the blood transfusion. Igs were low (IgM, $0.040 \mathrm{~g} / \mathrm{l} ; \mathrm{IgG}, 4.81 \mathrm{~g} / \mathrm{l} ; \mathrm{IgA}, 0.04 \mathrm{~g} / \mathrm{l} ; \mathrm{IgE}, 164 \mathrm{~g} / \mathrm{l}$ ), and proliferative response to mitogens was absent. While planning for an HSC transplan- tation was under way, she developed a severe respiratory complication and died of heart failure. Postmortem examination demonstrated a disseminated CMV infection. Molecular analysis of the RAG1 gene demonstrated compound-heterozygous mutations: 1 allele carried a duplication of 19 nucleotides from 2,020 to 2,038 , leading to frameshift and premature termination, while in the other allele the 368-369 dinucleotide deletion was detected. Approval for this study was obtained from the Ethical Committee of Spedali Civili (Brescia, Italy). Informed consent was obtained according to the World Medical Association Declaration of Helsinki.

Immunohistochemistry. Thin cryostat sections of human thymi obtained during autopsies with informed consent from 1 Omenn syndrome patient (Pt1), $1 \mathrm{~T}^{-} \mathrm{B}^{-}$SCID patient (Pt3), and 2 control children who underwent cardiac thoracic surgery, were used for morphological and immunophenotypical studies. Histological data from Omenn syndrome patient Pt1 have been previously reported (9). A formalin-fixed paraffin-embedded tissue section was stained with $\mathrm{H} \& \mathrm{E}$ for morphological evaluation. Immunohistochemistry for AIRE was performed with a rabbit polyclonal anti-AIRE antibody (22) (1:50 dilution), by an indirect technique using a streptavidin-biotin complex and immunoperoxidase on acetone-fixed thymus sections. AIRE was revealed using the StrAviGen Multilink kit (BioGenex). Immunofluorescence staining of AIREtransfected COS cells demonstrates the typical cellular distribution of the protein into nuclear dots and in the microtubular network, while it does not stain COS cells transfected with vector. Comparative analysis of mRNA and protein expression by real-time PCR and immunocytochemistry in the myelomonocytic cell line OCT-4, treated with GM-CSF, demonstrates complete correspondence between AIRE protein and mRNA expression.

For control samples, mock and pcDNA3-AIRE-transfected COS7 cells were used in immunofluorescence; anti-AIRE Ab (1:20 dilution) was incubated overnight and detected using biotinylated swine anti-rabbit antibodies (DAKO Cytomation) followed by streptavidin-FITC. Doubleimmunofluorescence technique was used to colocalize AIRE expression on TECs. Anti-human keratin (anti-pankeratin, clone MNF116, 1:200 dilution; DAKO Cytomation) was revealed using a goat anti-mouse IgG1 Texas redconjugated antibody (Vector Laboratories Inc.).

Transfections. COS7 cells were transfected with human AIRE full-length cDNA subcloned in pcDNA3.1 (Invitrogen Corp.) with Lipofectamine (Invitrogen Corp.), according to the instruction manual. The AIRE cDNA was confirmed by direct automated sequence.

Real-time PCR. RNA was purified from whole frozen thymus using the guanidinium thiocyanate-phenol-chloroform method according to the instruction manual (RNAwiz; Ambion Inc.). One microgram of DNasetreated total RNA was used to synthesize the first strand of CDNA with the GeneAmp RNA PCR kit (Applied Biosystems). For real-time PCR analysis, Assays-on-Demand products (20x) and TaqMan Master Mix (2x) from Applied Biosystems were used, according to the instruction manual, to amplify AIRE, keratin-13, cytochrome P450 1a2, insulin, FABP, LT $\beta R$, and GAPDH genes. Reactions were run on ABI PRISM 7700 Sequence Detection System (Applied Biosystems) in a final volume of $25 \mu \mathrm{l}$ for 50 cycles. We analyzed the expression levels of AIRE, insulin, keratin-13, and LT $\beta$ R, and we normalized the results to GAPDH levels in each sample.

Statistical analysis. Comparison of values between normal donors and patients was performed where indicated by Student's $t$ test for unpaired data. Differences were defined as significant for $P$ values lower than 0.05 .

\section{Acknowledgments}

We are grateful to the families of the patients who have given their consent for this study. We also thank Alessandra Melone for pBSK-AIRE plasmid containing human AIRE full-length cDNA, 
Francesca Gentili and Simona Marrelli for technical assistance, and Antonietta Silini for kindly reviewing the manuscript. This work was supported by grants from the Ministero dell'Istruzione dell'Università e della Ricerca (MIUR) Cofin 2002 (to E. Mazzolari) and Cofin 2004 (to L.D. Notarangelo); from MIUR-Fondo per gli Investimenti della Ricerca di Base (FIRB; to L.D. Notarangelo and A. Villa); from the Centro per l'Innovazione Diagnostica e Terapeutica, MIUR; from Progetto Finalizzato Ministry of Health by Joint Program Consiglio Nazionale delle Ricerche-MIUR (Law 449/97); from Progetto Strategico Min Salute-Bambin Gesù 2002 and Min Salute-S. Raffaele 2003 (to L.D. Notarangelo); from the European Union (QLR-2000-01395) and Min Salute-Burlo 2003 (to R. Badolato); and from Telethon-Association Française contre les Myopathies (AFM) (GAT0203; to A. Fischer, L.D. Notarangelo, and A. Villa). This is manuscript no. 76 of the Genome 2000/Istituto Tecnologie Biomediche Project funded by Fondazione Cariplo.

Received for publication August 19, 2004, and accepted in revised form December 7, 2004.

Address correspondence to: Raffaele Badolato, Istituto di Medicina Molecolare "Angelo Nocivelli," Università di Brescia, c/o Spedali Civili, 25123 Brescia, Italy. Phone: 39-0303996282; Fax: 39-0303996059; E-mail: badolato@med.unibs.it.
1. Omenn, G.S. 1965. Familial reticuloendotheliosis with eosinophilia. N. Engl. J. Med. 273:427-432.

2. Ochs, H.D., Davis, S.D., Mickelson, E., Lerner, K.G., and Wedgwood, R.J. 1974. Combined immunodeficiency and reticuloendotheliosis with eosinophilia. J. Pediatr. 85:463-465.

3. Le Deist, F., et al. 1985. Deficit immunitaire mixte et grave avec hypereosinophilie. Arch. Fr. Pediatr. 42:11-14.

4. Facchetti, F., Blanzuoli, L., Ungari, M., Alebardi, O., and Vermi, W. 1998. Lymph node pathology in primary combined immunodeficiency diseases. Springer Semin. Immunopathol. 19:459-478.

5. Saint-Basile, G., et al. 1991. Restricted heterogeneity of $\mathrm{T}$ lymphocytes in combined immunodeficiency with hypereosinophilia (Omenn's syndrome). J. Clin. Invest. 87:1352-1359.

6. Rieux-Laucat, F., et al. 1998. Highly restricted human $\mathrm{T}$ cell repertoire in peripheral blood and tissue-infiltrating lymphocytes in Omenn's syndrome. J. Clin. Invest. 102:312-321.

7. Harville, T.O., Adams, D.M., Howard, T.A., and Ware, R.E. 1997. Oligoclonal expansion of $\mathrm{CD} 45 \mathrm{RO}+\mathrm{T}$ lymphocytes in Omenn syndrome. J. Clin. Immunol. 17:322-332.

8. Brooks, E.G., Filipovich, A.H., Padgett, J.W., Mamlock, R., and Goldblum, R.M. 1999. T-cell receptor analysis in Omenn's syndrome: evidence for defects in gene rearrangement and assembly. Blood. 93:242-250.

9. Signorini, S., et al. 1999. Intrathymic restriction and peripheral expansion of the T-cell repertoire in Omenn syndrome. Blood. 94:3468-3478.

10. Schwarz, K., et al. 1996. RAG mutations in human B cell-negative SCID. Science. 274:97-99.

11. Villa, A., et al. 1998. Partial V(D)J recombination activity leads to Omenn syndrome. Cell. 93:885-896.

12. Noordzij, J.G., et al. 2000. N-terminal truncated human RAG1 proteins can direct T-cell receptor but not immunoglobulin gene rearrangements. Blood. 96:203-209.

13. Fischer, A. 2004. Human primary immunodeficiency diseases: a perspective. Nat. Immunol. 5:23-30.

14. Aaltonen, J. 1997. An autoimmune disease, APECED, caused by mutations in a novel gene featuring two PHD-type zinc-finger domains. The Finnish-German APECED Consortium. Autoimmune Polyendocrinopathy-Candidiasis-Ectodermal Dystrophy. Nat. Genet. 17:399-403.

15. Nagamine, K., et al. 1997. Positional cloning of the APECED gene. Nat. Genet. 17:393-398.

16. Rosatelli, M.C., et al. 1998. A common mutation in Sardinian autoimmune polyendocrinopathycandidiasis-ectodermal dystrophy patients. Hum. Genet. 103:428-434.

17. Zuklys, S., et al. 2000. Normal thymic architecture and negative selection are associated with Aire expression, the gene defective in the autoimmunepolyendocrinopathy-candidiasis-ectodermal dystrophy (APECED). J. Immunol. 165:1976-1983.

18. Chin, R.K., et al. 2003. Lymphotoxin pathway directs thymic Aire expression. Nat. Immunol. 4:1121-1127.

19. Heino, M., et al. 2000. RNA and protein expression of the murine autoimmune regulator gene (Aire) in normal, RelB-deficient and in NOD mouse. Eur. J. Immunol. 30:1884-1893.

20. Anderson, M.S., et al. 2002. Projection of an immunological self shadow within the thymus by the aire protein. Science. 298:1395-1401.

21. Liston, A., Lesage, S., Wilson, J., Peltonen, L., and Goodnow, C.C. 2003. Aire regulates negative selection of organ-specific T cells. Nat. Immunol. 4:350-354

22. Kogawa, K., et al. 2002. Expression of AIRE gene in peripheral monocyte/dendritic cell lineage. Immunol. Lett. 80:195-198.

23. Bjorses, P., et al. 2000. Mutations in the AIRE gene: effects on subcellular location and transactivation function of the autoimmune polyendocrinopathycandidiasis-ectodermal dystrophy protein. Am. J. Hum. Genet. 66:378-392.

24. Heino, M., et al. 1999. Autoimmune regulator is expressed in the cells regulating immune tolerance in thymus medulla. Biochem. Biophys. Res. Commun. 257:821-825.

25. Boehm, T., Scheu, S., Pfeffer, K., and Bleul, C.C. 2003. Thymic medullary epithelial cell differentiation, thymocyte emigration, and the control of autoimmunity require lympho-epithelial cross talk via LTbetaR. J. Exp. Med. 198:757-769.

26. Villa, A., et al. 2001. V(D)J recombination defects in lymphocytes due to RAG mutations: severe immunodeficiency with a spectrum of clinical presentations. Blood. 97:81-88.

27. Liston, A., et al. 2004. Gene dosage-limiting role of Aire in thymic expression, clonal deletion, and organ-specific autoimmunity. J. Exp. Med. 200:1015-1026.

28. Sakaguchi, S. 2004. The orign of FOXP3-expressing $\mathrm{CD}^{+}$regulatory $\mathrm{T}$ cells: thymus or periphery. J. Clin. Invest. 112:1310-1312. doi:10.1172/ JCI200320274.

29. Sharfe, N., Dadi, H.K., Shahar, M., and Roifman, C.M. 1997. Human immune disorder arising from mutation of the alpha chain of the interleukin-2 receptor. Proc. Natl. Acad. Sci. U. S. A. 94:3168-3171.

30. Schmalstieg, F.C., et al. 1995. Missense mutation in exon 7 of the common $\gamma$ chain gene causes a moderate form of X-linked combined immunodeficiency. J. Clin. Invest. 95:1169-1173.

31. Santagata, S., et al. 2000. N-terminal RAG1 frameshift mutations in Omenn's syndrome: internal methionine usage leads to partial V(D)J recombination activity and reveals a fundamental role in vivo for the N-terminal domains. Proc. Natl. Acad. Sci. U. S. A. 97:14572-14577. 